\title{
HILL Jonathan D., Made-from-bone. Trickster myths, music, and history from the Amazon
}

\section{Emmanuel de Vienne}

\section{OpenEdition}

\section{Journals}

Édition électronique

URL : http://journals.openedition.org/jsa/11822

DOI : 10.4000/jsa.11822

ISSN : 1957-7842

\section{Éditeur}

Société des américanistes

\section{Édition imprimée}

Date de publication : 5 octobre 2011

Pagination : 369-372

ISSN : 0037-9174

\section{Référence électronique}

Emmanuel de Vienne, « HILL Jonathan D., Made-from-bone. Trickster myths, music, and history from the Amazon », Journal de la Société des américanistes [En ligne], 97-1 | 2011, mis en ligne le 10 juillet 2011 , consulté le 24 septembre 2020. URL : http://journals.openedition.org/jsa/11822 ; DOI : https://doi.org/ $10.4000 /$ jsa. 11822

Ce document a été généré automatiquement le 24 septembre 2020

(c) Société des Américanistes 


\title{
HILL Jonathan D., Made-from-bone. Trickster myths, music, and history from the Amazon
}

\author{
Emmanuel de Vienne
}

\section{RÉFÉRENCE}

HILL Jonathan D., Made-from-bone. Trickster myths, music, and history from the Amazon, University of Illinois Press, Urbana/Chicago, 2009, xxii + 195 p., bibl, gloss., index, ill., cartes, photos

1 Toute mythologie ne se lit pas comme un roman. C'est bien l'intérêt et le succès de ce livre que d'être à ce titre une exception. Pour parvenir à ce résultat que tout ethnographe sait ardu, Jonathan Hill s'est donné en apparence un objectif modeste sur le plan théorique, celui de «fournir un ensemble complet de traductions anglaises des récits Wakuénai sur le passé mythique et ses transformations » (p. i). Dans ce groupe de langue arawak du haut río Negro, la figure mythique centrale est Made-from-Bone, un trickster qui peut compter pour triompher de ses ennemis sur une capacité inégalée à lire les intentions et à manipuler le langage et plus généralement les signes. Le plaisir de lecture tient en grande partie à cette impression d'un ensemble complet, ou du moins organisé. La mythologie wakuénai s'articule en effet en trois parties : « les temps primordiaux », «le monde commence » et «le monde s'élargit » dont Hill montre les spécificités et les rapports qu'elles entretiennent entre elles. La première partie est caractérisée par une violence effective ou potentielle entre affins, tout en commençant d'ailleurs par le meurtre d'un homme par son beau-frère (WB) Great-Sickness. Les os des doigts du mort, conservés par sa veuve, deviennent deux frères-Criquets, dont l'aîné est Made-from-Bone. Ces deux frères doivent contrer les velléités homicides de leur oncle. Puis, dans les mythes qui suivent, Made-from-Bone passe l'essentiel de son temps à éviter d'être tué par ses beaux-pères, beaux-frères et épouses. Sa capacité à 
tromper augmente d'ailleurs graduellement, pour culminer dans le mythe d'origine des mauvais présages. En attachant des interprétations de plus en plus graves à une série de signes (mauvais rêves, sons étranges, odeur, chute, oiseau inhabituel, etc.), il développe une "méthode de terreur sémiotique " qu'il met à profit contre sa femme qui avait tenté de l'assassiner avec l'aide de son frère.

2 Dans la deuxième partie, Made-from-Bone obtient par la ruse des biens et des pratiques auprès de différents êtres mythiques: le sommeil et la nuit, le feu et les palmiers. La violence a cédé la place à une quête pour préparer et créer tout ce dont auront besoin «les nouveaux gens du monde futur ». Made-from-Bone cesse ici d'être le seul héros culturel. Son jeune frère, Manioc-Man, est le protagoniste d'un récit qui explique le passage de l'hostilité entre affins (propre aux temps primordiaux) à des échanges pacifiques et fondés sur le respect et la réciprocité. Remarié après avoir été trompé par sa première épouse, Manioc-Man enseigne aux enfants de son second lit comment demander par le chant rituel mádzukerái la nourriture et les boissons fermentées. Cette demande ritualisée chantée est l'inverse de la violence qui a cours dans la première partie du cycle rituel.

3 La troisième partie, "le monde s'élargit ", met en scène Kuwái, fruit de l'union incestueuse de Made-from-Bone avec sa tante paternelle, Première-Femme. Créateur des rituels de fabrication qui rythment le cycle de vie, Kuwái meurt par le feu à l'instigation de Made-from-Bone. De ses cendres naît un arbre dont il redescend sous forme de flûtes et de trompettes, qui sont dès lors l'objet d'une querelle entre les femmes (sous la conduite de Première-Femme) et les hommes (guidés par Made-fromBone). «Dans leur lutte collective pour contrôler les flûtes et les trompettes de Kuwái, les hommes et les femmes jouent une musique qui crée et élargit le monde pour en faire cette immense région de forêt et de rivières habitée aujourd'hui par les hommes » (p.115). Cette section rassemble aussi les mythes d'origine du chamanisme et de la sorcellerie.

4 Si ce livre n'était qu'une traduction éclairée de cette mythologie, on pourrait lui reprocher l'absence de transcription en langue indigène, de juxtalinéarisation et d'analyse linguistique, même ponctuelle. Mais son projet est surtout ethnologique : il s'agit en vérité de montrer la fécondité d'une approche qui privilégie la compréhension du rapport entre mythe et histoire (Claude Lévi-Strauss n'apparaît d'ailleurs pas dans la bibliographie). De ce point de vue, loin d'être une affirmation consensuelle, son projet d'envisager la mythologie «non comme un reliquat folklorique d'un passé indigène intouché, mais comme une pratique symbolique dynamique d'interprétation et d'engagement dans un monde contemporain que nous partageons tous » (p. xix) trouve ici une illustration convaincante et précise. Le premier chapitre donne ainsi une synthèse de l'histoire des Wakuénai, dans un contexte géographique nécessairement large, celui du Nord et du Nord-Ouest amazoniens. Le vaste réseau d'échanges auquel les ancêtres des Wakuénai participaient avant la Conquête s'est trouvé largement affecté par les épidémies, les missions et l'esclavage durant la période coloniale. Situés entre deux forces opposées (les Hollandais alliés aux Caribes au nord, les commerçants portugais au sud), ils ont dû pour survivre, tout au long des XVII et XVIII ${ }^{e}$ siècles, à la fois fuir et participer à des confédérations interethniques pour faire face à des menaces ponctuelles. La violence connaît un nouveau sommet avec le boom du caoutchouc, en particulier au début $\mathrm{du} \mathrm{xx}^{\mathrm{e}}$ siècle, lorsque Tomas Funes entreprend des raids génocidaires systématiques. Enfin, le contexte de la première enquête de Hill en 
1980-1981 est mis en perspective avec la christianisation brutale conduite par les missionnaires des New Tribes à partir des années 1950. Hill va plus loin qu'une mise en rapport de la violence qui caractérise les temps primordiaux avec celle qui marque sur près de quatre siècles l'histoire de la région. Il procède de manière systématique par des « interludes ", à la fin de chaque partie, dans lesquels un mythe en particulier est analysé à la lumière de l'histoire ou de l'ethnographie.

5 À la fin de la première partie, l'auteur propose ainsi en premier interlude une analyse ethnohistorique du mythe racontant la grossesse adultérine de l'épouse de Made-fromBone. Dans ce mythe, le trickster créateur découvre que sa femme le trompe avec Anaconda, et décide de se venger. Hill montre que l'Anaconda est ici une «métaphore historique " de l'histoire coloniale, capable de renvoyer aussi bien aux rapports entre Wakuénai et Blancs qu'entre groupes arawak et tukano durant cette période.

6 L'interlude ethnomusicologique qui clôt la deuxième partie se propose d'éclairer le mythe d'origine du rituel d'échange cérémoniel pudáli par le récit d'une occurrence observée en 1981 au cours de laquelle les flûtes kulirrína ont été réintroduites après des décennies d'absence. Bien écrite et intéressante parce qu'elle ne masque pas le contexte de son organisation (en particulier la place centrale occupée par l'ethnologue-mécène), cette partie pèche cependant peut-être par sa brièveté. Le sous-genre vocal mádzurekái, en particulier, par lequel on adresse des demandes chantées de boisson et de biens, n'est illustré que par deux traductions. Si celles-ci suffisent à saisir la logique ironique qui préside à la composition de ces chants, elles sont peut-être insuffisantes au regard de l'importance dans la mythologie de ce moment qui opère le passage entre la violence des temps primordiaux et l'échange pacifique du « monde qui commence ».

7 La coda qui clôt l'ouvrage s'intéresse au dernier mythe de la partie «le monde s'élargit ». Celui-ci (The origin of enchanted spirits and the city of gold) raconte le voyage d'un homme orphelin dans la ville très moderne et où l'or abonde de Temedawí, qui appartient aux esprits yopinái. En plus d'y épouser une femme, selon un motif classique, le héros rencontre le "président " de la ville qui le nomme docteur en charge de l'hôpital pour le restant de ses jours. Ce mythe est éclairé par les changements des rapports avec l'État dans la décennie 1990, qui voit une lutte pour la reconnaissance des droits des communautés autochtones. Mettant en relation ce récit avec l'essor du consumérisme, de l'individualisme et de l'alphabétisation, Hill y lit également une reconfiguration des rapports entre chamanisme et État.

8 Sans souscrire systématiquement aux analyses de l'auteur, on reste convaincu de la fécondité d'une approche historique, en particulier lorsqu'elle concerne le présent: sans cesser d'être par définition conjecturale, elle se nourrit alors de faisceaux d'éléments suffisamment précis pour emporter l'adhésion. On peut néanmoins regretter - mais ce n'est pas là le but du livre - un manque de description des usages ou des effets du mythe. La préface donne de manière précise le contexte de production de l'ouvrage : un premier corpus est enregistré ou recueilli en 1980, 1981 et 1985 auprès d'Horacio López Pequeira. Celui-ci décède en 1991, et c'est donc son fils Felix López Oliveiros qui aide Hill en 1998 à élaborer, six mois durant, les transcriptions et les traductions en espagnol pour un projet de publication bilingue soutenu par le ministère de l'Éducation. On reconnaît ici un contexte désormais répandu, qui tend parfois à supplanter les énonciations quotidiennes. Mais cela n'empêchait pas de préciser ce que Felix Oliveiros, dans ce labeur commun, a formulé comme explications et commentaires aux récits. Dans la mesure en particulier où Hill cherche parfois à dégager la leçon ou la 
morale des histoires, il est dommage qu'il ne s'appuie pas davantage sur la parole des Wakuénai. De plus, que Felix soit peut-être le premier narrateur du mythe de la ville d'or laisse un certain nombre de questions en suspens : qu'en est-il des modalités de légitimation du mythe lorsque son contenu est manifestement nouveau? Même en l'absence de réponses, on ne peut que conseiller la lecture de cet excellent recueil.

\section{AUTEURS}

\section{EMMANUEL DE VIENNE}

Laboratoire d'anthropologie sociale 